\title{
LA DISPERSIÓN DE LOS REGÍMENES LABORALES EN EL SECTOR PÚBLICO LIMITAN LA REMUNERACIÓN HOMOLOGADA Y \\ LOS BENEFICIOS DE LOS DOCENTES DE LA UNMSM
}

\author{
DISPERSION OF SCHEMES IN THE PUBLIC SECTOR LABOR LIMIT AS SALARIES \\ APPROVED AND BENEFITS OF TEACHERS OF UNMSM

\section{Esteban Marino Avelino SÁnchez *} \\ Docente Asociado de la Facultad de Ciencias Contables \\ Jeri Gloria Ramón Ruffner ** \\ Docente Principal de la Facultad de Ciencias Contables
}

Universidad Nacional Mayor de San Marcos-UNMSM / Lima-Perú

[Recepción: Julio de 2013/ Conformidad: Agosto 2013]

\section{RESUMEN}

El problema es el incumplimiento reiterado del gobierno peruano de sus propias disposiciones legales sobre montos remunerativos de los magistrados del Poder Judicial y, especialmente, de los docentes de las universidades públicas, lo que genera malestar, más aún por la aplicación paralela de los regímenes laborales en el sector privado que se administra como público pero cuesta como privado. La remuneración mensual de libre disposición de los congresistas en los últimos 15 años fue mayor a S/.23 000.00; la remuneración de los jueces superiores y de primera instancia es menor de lo que dispone el artículo $186^{\circ}$ de la Ley Orgánica del Poder Judicial; la remuneración homologada de los docentes de la Universidad Nacional Mayor de San Marcos - UNMSM representa menos de la tercera parte de los ingresos de los magistrados de los niveles con los cuales se homologó. Los montos de beneficios sociales calculados con las normas del Reglamento del Congreso contrastan con los montos calculados con las normas del Decreto Legislativo 276; las diferencias son abismales, la CTS de los jueces supremos es 396 veces más con respecto a la CTS de los docentes principales, porque no existe una política de recursos humanos clara y acorde con los objetivos de desarrollo económico y social nacional.

\section{Palabras Claves:}

Empleo y remuneraciones en las universidades públicas; homologación de remuneraciones de docentes universitarios; remuneración de congresistas; remuneración de magistrados del Poder Judicial; remuneración computable para cálculo de CTS.

\begin{abstract}
The problem is the repeated failure of the Peruvian government of its own law on remunerative amounts of magistrates of the judiciary and especially of teachers of public universities, which creates discomfort, indeed by the parallel application of labor regimes in the private sector, but is administered as public and private costs. The monthly remuneration received, freely available, of Congress in the last 15 years was higher than $\mathrm{S} / .23000 .00$, remuneration of senior judges and trial is less than what is stated in Article 186 of the Law on the Judiciary, the approved remuneration of teachers in the San Marcos National University - UNMSM represents less than a third income of the judges of the levels with which was approved. The social benefit amounts calculated with the rules of Congress Rules contrast with the amounts calculated with the rules of the Legislative Decree 276, the differences are abysmal, the supreme judges CTS 396 times compared to the CTS primary teachers because there is no clear human resources policy and objectives in line with national economic and social development.
\end{abstract}

\section{Keywords:}

Employment and wages in public universities, approval of remuneration of university professors, congressmen compensation, compensation of judges of the Judiciary, calculating compensation for CTS computable.

* Magister en Contabilidad con Mención en Auditoría. Contador Público y Economista Colegiado. Email: estebanavelino@gmail.com

** Doctora en Contabilidad. Magister en Contabilidad con mención en Auditoría. Contadora Pública Colegiada- UNMSM. Email: jeriramon_162@hotmail.com 


\section{INTRODUCCIÓN}

En este artículo, recordamos los regímenes laborales en el Perú, vigentes más de 20 años y sus consecuencias, específicamente, en tres entidades del sector público: el Congreso de la República; el Poder Judicial y la UNMSM teniendo como objetivo analizar las normas legales sobre empleo, remuneraciones y beneficios sociales de los trabajadores de la Universidad Nacional Mayor de San Marcos. Planteamos como hipótesis: "La remuneración de los docentes de las universidades públicas, antes y después de la homologación, comparada con las remuneraciones de los magistrados del Poder Judicial son diminutas, respecto a los ingresos de los mismos magistrados, los profesionales del Congreso y funcionarios de confianza de otros sectores de la administración pública, de categorías equivalentes"; se demuestra analizando los regímenes laborales y las estadísticas de empleo y de remuneraciones de las entidades aludidas.

En este artículo, aplicamos los métodos cualitativo y descriptivo de las normas legales pertinentes como las técnicas: observación, selección y clasificación de información, cruce y análisis de información facilitadas (a nuestro requerimiento) por el Congreso de la República; el Poder Judicial y la Universidad Nacional Mayor de San Marcos. Presentamos en forma sucesiva: el marco conceptual, antecedentes, regímenes laborales, beneficios sociales, empleo, remuneraciones y las conclusiones.

Tres trabajos de investigación son referenciados como antecedentes del problema abordado; primero, el Informe sobre la Situación del Personal de la Administración Pública Central publicado en Separata Especial, en el Diario El Peruano de fecha 25/07/2001, de la Comisión Multisectorial creada por Decreto Supremo No 004-2001-TR, donde los problemas revelados once años después, continúan aún sin solución. El segundo, el Estudio sobre Recursos Humanos en Salud, que también analiza la formación universitaria de profesionales de la salud de GRADE, donde señala haberse encontrado un marco normativo fragmentado y disperso de normas de diverso origen con objetivos superpuestos y poco transparente. El tercero: Los Regímenes Laborales en el Perú de SERVIR, donde se informa que de los quince regímenes laborales, tres son regímenes generales; nueve son regímenes especiales, uno de gerentes públicos y otro sin carrera con vínculo civil -convenios de administración con PNUD- y el Fondo de Apoyo Gerencial, creado por Decreto Ley $\mathrm{N}^{\circ}$ 25650 en 1992.

Los estudios señalados en el numeral 2 no son referidos específicamente a la universidad. Sin embargo, la legislación de los regímenes laborales generales, analizada en los mismos, también se aplica en las universidades públicas. Dichos estudios revelan la dispersión absurda de regímenes laborales $y$ de montos remunerativos en entidades del sector público y que inducen a conflictos laborales latentes. Por estas consideraciones, los estudios comentados, se vinculan estrechamente con nuestro trabajo de investigación, el que postula dar cuenta del mismo problema en la UNMSM y en el Poder Judicial, lo que constituye nuestro aporte.

El propósito que nos motivó a realizar este estudio, es demostrar que la "remuneración mensual homologada" de los docentes de la UNMSM sigue siendo diminuta respecto a las remuneraciones que reciben los funcionarios de confianza del Congreso de la República y magistrados del Poder Judicial con formación profesional y estudios de posgrado equivalentes. Como se podrá observar más adelante, la "remuneración homologada" de los docentes de la Universidad Nacional Mayor de San Marcos representa menos del $30 \%$ de los ingresos mensuales de los magistrados del Poder Judicial. En beneficios sociales, las diferencias son abismales, debido a que no existe una política de recursos humanos del Gobierno clara y acorde con los objetivos de desarrollo económico y social.

\section{MARCO CONCEPTUAL}

¿Qué es el empleo? Entiéndase como ocupación, tarea u oficio que realizan las personas con la finalidad de obtener bienes para asegurar su bienestar económico, social y cultural. 
LA DISPERSIÓN DE LOS REGÍMENES LABORALES EN EL SECTOR PÚBLICO LIMITAN LA REMUNERACIÓN HOMOLOGADA Y LOS BENEFICIOS DE LOS DOCENTES DE LA UNMSM

Remuneración. Es la compensación económica que recibe el trabajador por su trabajo prestado a una empresa o institución, destinada para su subsistencia como de su familia; también se dice que es la retribución directa e indirecta al trabajador por su trabajo prestado a un empleador. La retribución directa es el pago que recibe el trabajador en forma de sueldos, salarios, primas y comisiones; la retribución indirecta, llamada también beneficios, se otorga por derechos y prestaciones que se adquieren como consecuencia de la relación laboral, como: vacaciones, gratificaciones, asignación familiar, seguros, etc. Las remuneraciones y compensaciones se otorgan a los trabajadores por sus servicios prestados, pudiendo ser esfuerzos físicos o mentales.

De conformidad con el artículo $6^{\circ}$ del Texto Único Ordenado del Decreto Legislativo No 728, Ley de Productividad y Competitividad Laboral, aprobado por el Decreto Supremo No 003-97-TR: "Constituye remuneración para todo efecto legal el íntegro de lo que el trabajador recibe por sus servicios, en dinero o en especie, cualquiera sea la forma o denominación que tenga, siempre que sean de su libre disposición”. El artículo $7^{\circ}$ de la misma ley dice: “No constituye remuneración para ningún efecto legal los conceptos previstos en los artículos $19^{\circ}$ y $20^{\circ}$, (las gratificaciones extraordinarias -entre otros- y la alimentación proporcionada por el empleador en calidad de condición de trabajo) del TUO del Decreto Legislativo $\mathrm{N}^{\circ} 650$.

\section{ANTECEDENTES}

El "Informe sobre la Situación del Personal de la Administración Pública Central” de la Comisión Multisectorial - CM (creada por D.S. N 004-2001TR), publicado en la Separata Especial en el Diario El Peruano de fecha 25/07/2001, menciona que la remuneración mensual de los funcionarios de "alta dirección” entiéndase Ministros de Estado y sus asesores, al final del Gobierno de Fujimori, fluctúo entre S/.35 600.00yS/.44711.00, de los funcionarios F-5 entre $S / .1912 .00$ y $S / .12438 .00$ y de los profesionales SPA entre S/. 835.00 y S/. 4 980.00. El "Informe Final de Estudio sobre los Recursos Humanos en Salud en el Perú ( ... )” de GRADE (año 2006), sostiene: "No existe una política de recursos humanos

\section{Tabla $\mathbf{N}^{\circ} 1$ \\ Ingresos mensuales promedio de los servidores públicos del año 2012 (Nuevos Soles)}

\begin{tabular}{|c|c|c|c|c|}
\hline \multirow{2}{*}{$\begin{array}{c}\text { Entidad u Organismo } \\
\text { Público }\end{array}$} & \multicolumn{2}{|c|}{ Decreto Legislativo 276} & \multicolumn{2}{|c|}{ Decreto Legislativo 728} \\
\hline & Ingreso mínimo & Ingreso máximo & Ingreso mínimo & Ingreso máximo \\
\hline Ministerios 1/ & 400 & 15600 & 600 & 15600 \\
\hline OCA 2/ & 2137 & 27117 & 1000 & 32560 \\
\hline O. P. Reguladores & - & - & 1364 & 15600 \\
\hline Técnicos Especializados & 841 & 12270 & 1400 & 15660 \\
\hline O. P. Ejecutores $3 /$ & 528 & 10395 & 273 & 15951 \\
\hline $\begin{array}{ll}1 / & \text { No considera Ministerio de Edu } \\
2 / & \text { Organismos Constitucionalmen } \\
3 / & \text { Sobre una muestra de } 5 \text { Organ }\end{array}$ & 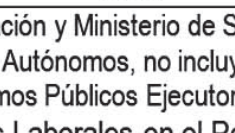 & , & & \\
\hline
\end{tabular}

clara y acorde con objetivos definidos".

"Los Regímenes Laborales en el Perú" de SERVIR publicado en su pág. web; informa de quince regímenes laborales en las entidades del Estado, con obligaciones similares y distintos derechos: tres regímenes generales (Decretos Legislativos 276, 728 y1057, de los años 1984, 1991 y 2012, respecti- vamente), nueve regímenes especiales (Magisterial -Ley 24029 -año 1984-, Docentes Universitarios -Ley 23733 -año 1983-, etc.), sin carrera: uno con vínculo laboral (gerentes públicos -Decreto Legislativo 1024 del año 2008) y otro, con vínculo civil (convenios de administración con PNUD) y el Fondo de Apoyo Gerencial (Decreto Ley 25650 del año 1992). Exclu- 
yendo a los altos funcionarios y a las bonificaciones especiales, las remuneraciones en el sector público en los últimos años fluctúan como se aprecia en la tabla $\mathrm{N}^{\circ} 1$.

\section{REGÍMENES LABORALES Y DE BENEFICIOS SOCIALES}

Los congresistas, conforme al artículo 21 del Reglamento del Congreso - $\mathrm{RC}$, son funcionarios públicos al servicio de la nación, tienen derecho a la seguridad social en materia de salud y pensiones, conforme a los Decretos Leyes 20530, 19990, 19846 y 21021, según el régimen al que pertenezca, y en base al derecho pensionario que tenía al ingresar al Congreso; no están comprendidos en la "Ley de la Carrera Administrativa", salvo en lo que les fuera aplicable. Reciben una "remuneración adecuada" sujeta al pago de los tributos de ley y a una compensación por tiempo de servicios, calculada con dicha remuneración; adicionalmente, tienen derecho a la contratación de seguros privados para ellos y sus familiares dependientes. Los funcionarios y trabajadores del Congreso, conforme lo dispone el Reglamento del Congreso, están comprendidos en el régimen laboral privado del 728 .

Los magistrados del Poder Judicial están comprendidos en la Ley 29277 -Ley de la Carrera Judicial, así como, en la Ley Orgánica del Poder Judicial; sus remuneraciones por todo concepto, de acuerdo con los literales a) yb) del numeral 5 del artículo $186^{\circ} \mathrm{de}$ la LOPJ y normas modificatorias, son los porcentajes de la remuneración de los Congresistas de la República, que se indican en la tabla $\mathrm{N}^{\circ} 2$.

Tabla $\mathrm{N}^{\circ} 2$

Remuneraciones de los Magistrados del Poder Judicial (Porcentajes de la remuneración de un Congresista -RC)

\begin{tabular}{|c|c|c|}
\hline Nivel del magistrado & Artículo 186 de la LOPJ & Artículo 4 de la Ley $28212\left(^{*}\right)$ \\
\hline Jueces Supremos & $100 \%$ de la RC & 6 URSP $(100 \%$ de la RC) \\
\hline Jueces Superiores & $90 \%(+)$ & $81 \%(+)$ \\
\hline Jueces especializados o Mixtos & $80 \%(+)$ & $58 \%(+)$ \\
\hline Jueces de Paz Letrados & $70 \%(+)$ & $40 \%(+)$ \\
\hline Secretarios y Relatores de Sala & $55 \%(+)$ & No dice nada sobre este nivel \\
\hline \multicolumn{3}{|c|}{$\begin{array}{l}\text { Ley Orgánica del Poder Judicial, } \\
\text { Unidad remunerativa del sector público } \\
\text { Ley de ingresos de los altos funcionarios y autoridades del Estado, modificada por la Ley No } \\
29718 \text { (Diario, El Peruano: } 25 / 06 / 2011 \text { ) y suspendida su aplicación por la Ley No } 29818 .\end{array}$} \\
\hline \multicolumn{3}{|c|}{$\begin{array}{l}\text { Respecto de la remuneración de los jueces supremos. } \\
\text { Avelino, Esteban (2013). "Diagnóstico de Empleo y Remuneraciones en las Universidades Públicas } \\
\text { (Caso UNMSM)" - Proyecto de investigación SIN - SIN. }\end{array}$} \\
\hline
\end{tabular}

Seis meses después de publicada la Ley 29718, el 24 de diciembre de 2011 se publicó la Ley 29818, mediante la cual se dispone la constitución de una comisión conformada por representantes del Poder Judicial y del Ministerio de Economía y Finanzas, con el fin de evaluar la situación de los ingresos de los jueces a que se refiere el artículo $1^{\circ}$ de la Ley $N^{\circ} 29718$ (que modificó el artículo $4^{\circ}$ de la Ley $\mathrm{N}^{\circ} 28212$ ); con el objeto de proponer un proyecto de ley dentro del plazo de 180 días calendario. Se dejó en suspenso, en todos sus efectos, la aplicación de la Ley 29718 y en consecuencia, se restituyó la vigencia del artículo $4^{\circ}$ de la Ley $\mathrm{N}^{\circ} 28212$ y el artículo $186^{\circ}$ de la Ley Orgánica del Poder Judicial. La comisión aludida trabajó -de enero a setiembre del año 2012- el proyecto de ley financiada; pero el MEF en octubre del mismo año, dio a conocer su oposición al proyecto aludido.

Los magistrados del Poder Judicial, de acuerdo con los literales c, d y e del numeral 5 del artículo $186^{\circ}$ de la Ley Orgánica del Poder Judicial, perciben 16 haberes mensuales al año (por motivo de Vacaciones, Navidad, Escolaridad y Fiestas Patrias); los

12/ QVIPUKAMAYOC | Vol. 21(40) 2013 
LA DISPERSIÓN DE LOS REGÍMENES LABORALES EN EL SECTOR PÚBLICO LIMITAN LA REMUNERACIÓN HOMOLOGADA Y LOS BENEFICIOS DE LOS DOCENTES DE LA UNMSM

magistrados supremos al jubilarse siguen gozando de sus derechos adquiridos con arreglo a ley; los magistrados que queden inhabilitados para el trabajo, con ocasión del servicio judicial, perciben como pensión el íntegro de la remuneración que corresponda a su nivel. En caso de muerte el cónyuge e hijos perciben como pensión el haber que corresponde al grado inmediato superior (Única Disposición Complemen- taria y Modificatoria de la Ley No 29718, publicada el 25 de junio 2011). Su CTS se calcula agregando a la remuneración principal toda otra cantidad que perciban en forma permanente.

Los funcionarios y servidores públicos del Poder Judicial están comprendidos en los Decretos Legislativos 276, 728 y 1057 (del CAS).

Tabla $\mathbf{N}^{\circ} 3$

Remuneración Homologada de Docentes de Universitarios Públicas con la de los Magistrados del PJ

(Porcentajes de la remuneración de los vocales supremos)

\begin{tabular}{|c|c|c|}
\hline Categoría docente & Nivel del magistrado & Remuneración \\
\hline Principal a TC & Juez Supremo & $100 \%$ de la RC $\left(^{*}\right)$ \\
\hline Asociado a TC & Juez Superior & $90 \%\left(^{*}\right)$ \\
\hline Auxiliar a TC & Juez de Primera Instancia & $80 \%\left(^{\star}\right)$ \\
\hline
\end{tabular}

Fuente: Avelino, Esteban (2013). "Diagnóstico de Empleo y Remuneraciones en las Universidades Públicas (Caso UNMSM)" - Proyecto de investigación SIN - SIN.

Los docentes de las universidades públicas están comprendidos en la Ley $\mathrm{N}^{\circ} 23733$-Ley Universitaria y Decreto Legislativo 276 (para beneficios sociales). Sus haberes se "homologan" con las remuneraciones de los magistrados judiciales, dice el artículo $53^{\circ}$ de la misma Ley. Los trabajadores no docentes de la UNMSM están comprendidos en los Decretos Legislativos 276 y 1057 (del CAS). Los docentes y trabajadores administrativos de la UNMSM reciben los beneficios sociales establecidos en el Decreto Legislativo 276 y ley del CAS.

El 4 de julio de 2013 se publicó la Ley 30057, Ley del Servicio Civil, con la cual los sistemas remunerativos en el sector suman 16 ( $\sin$ incluir la ley de altos funcionarios del Estado), la que ha suscitado mucha oposición de los trabajadores del sector público porque no resolvería el problema de fondo: la racionalidad de una escala remunerativa e igual remuneración para trabajos equivalentes, con ello los regímenes especiales no tendrían razón de existir.

\section{EMPLEO Y REMUNERACIONES}

La remuneración total mensual de los congresistas (incluyendo los gastos operativos) en las últimas décadas, prácticamente, se mantiene constante, en algo más de 23 mil nuevos soles. Las remuneraciones de los funcionarios y servidores del Congreso se indican la tabla $\mathrm{N}^{\circ} 4$. 
Tabla $N^{\circ} 4$

Remuneración mensual de Funcionarios y Servidores del Congreso de la República (Nuevos Soles)

\begin{tabular}{|c|c|c|c|c|c|c|}
\hline \multirow{2}{*}{$\begin{array}{c}\text { Grupo } \\
\text { Ocupacional }\end{array}$} & \multicolumn{2}{|c|}{2005} & \multicolumn{2}{c|}{2010} & \multicolumn{2}{c|}{2012} \\
\cline { 2 - 7 } & R. Mínima & R. Máxima & R. Mínima & R. Máxima & R. Mínima & R. Máxima \\
\hline F & 10012 & 15326 & 10012 & 15326 & 10012 & 15326 \\
\hline SP & 3451 & 8899 & 4275 & 11021 & 4275 & 11021 \\
\hline ST & 1998 & 4697 & 1998 & 7189 & 1998 & 7189 \\
\hline SA & 1100 & 3069 & 1473 & 3069 & 1473 & 3069 \\
\hline
\end{tabular}

Fuente: $\quad$ Avelino, Esteban (2013). "Diagnóstico de Empleo y Remuneraciones en las Universidades Públicas (Caso UNMSM)" - Proyecto de investigación SIN - SIN.

La remuneración total mensual (incluido los gastos operativos) de los magistrados del Poder Judicial, en el mismo periodo se duplicó, como se observa en la tabla 5. Sin embargo, el MEF continúa contrariando más de 20 años lo dispuesto en el literal b) del numeral 5 del artículo $186^{\circ}$ de la LOPJ; asimismo con el numeral 1 del artículo $118^{\circ}$ de la Constitución.
En el mismo periodo, la remuneración promedio de los trabajadores del Poder Judicial comprendidos en el D.L. 276 aumentó 30\%, pero no sobrepasa los S/.1 830.00 mensual. Este gasto no es representativo porque dichos trabajadores representan menos del $1 \%$ del total de trabajadores del Poder Judicial.

\section{Tabla $\mathrm{N}^{\circ} 5$}

Número de Magistrados del Poder Judicial y Remuneración mensual (Nuevos Soles)

\begin{tabular}{|l|r|c|r|r|r|c|}
\hline \multirow{2}{*}{ Nivel del Magistrado } & \multicolumn{2}{|c|}{2000} & \multicolumn{2}{|c|}{2005} & \multicolumn{2}{c|}{2012} \\
\cline { 2 - 7 } & \multicolumn{1}{|c|}{$\mathrm{N}^{\circ}$} & Remuneración & \multicolumn{1}{c|}{$\mathrm{N}^{\circ}$} & Remuneración & \multicolumn{1}{c|}{$\mathrm{N}^{\circ}$} & Remuneración \\
\hline Juez Supremo & 41 & 12364.79 & 41 & 26094.79 & 38 & 23217.20 \\
\hline Juez Superior & 477 & 5705.07 & 486 & 12005.07 & 724 & 12005.07 \\
\hline Juez Especializado & 811 & 4305.07 & 875 & 9005.07 & 1358 & 9005.07 \\
\hline Juez de Paz & 375 & 3245.05 & 437 & 6205.05 & 607 & 6205.05 \\
\hline Total & 1704 & & 1839 & & 2727 & \\
\hline
\end{tabular}

Fuente: Avelino, Esteban (2013). "Diagnóstico de Empleo y Remuneraciones en las Universidades Públicas

(Caso UNMSM)" - Proyecto de investigación SIN - SIN.

El número de magistrados del Poder Judicial entre los años 2000 y 2012 aumentó más de 1000. Sin embargo, el aumento de la carga procesal -en el mismo periodo- habría sido mayor, lo que explicaría el crecido número de inculpados detenidos varios años, sin sentencia.

El total de docentes ordinarios y contratados de la UNMSM en la última década superó los 3100. Su remuneración en los últimos años, aumentó como consecuencia de la homologación, como se observa en la tabla $\mathrm{N}^{\circ} 6$. Sin embargo, la "remuneración homologada" de los Docentes Principales de la UNMSM es menos del 30\% de la remuneración mensual total de los Jueces Supremos, en las categorías Asociado y Auxiliar, prácticamente, el 25\% de la remuneración de los magistrados de los niveles con los cuales se homologó.

El número de trabajadores no docentes de la UNMSM, aunque en el año 2010 disminuyó, prácticamente se mantiene mayor a 3000 . Los contratos 
LA DISPERSIÓN DE LOS REGÍMENES LABORALES EN EL SECTOR PÚBLICO LIMITAN LA REMUNERACIÓN HOMOLOGADA Y LOS BENEFICIOS DE LOS DOCENTES DE LA UNMSM

\section{Tabla $\mathrm{N}^{\circ} 6$ \\ Número de Docentes Ordinarios por Categoría de la UNMSM y sus Remuneraciones (Nuevos Soles)}

\begin{tabular}{|l|r|r|r|r|r|r|}
\hline \multirow{2}{*}{ Categoria } & \multicolumn{2}{|c|}{ Empleo } & \multicolumn{2}{c|}{2010} & \multicolumn{2}{c|}{2011} \\
\cline { 2 - 7 } & 2002 & 2005 & Empleo & Remuneración & Empleo & Remuneración \\
\hline Principal & 492 & 553 & 725 & 3398,95 & 742 & 6707,32 \\
\hline Asociado & 1163 & 1170 & 1287 & 2274,69 & 1248 & 3008,00 \\
\hline Auxiliar & 776 & 821 & 899 & 1549,48 & 941 & 2008,00 \\
\hline Jefe de Práctica & 121 & 99 & 99 & 996,18 & 61 & 996,18 \\
\hline \multicolumn{1}{|c|}{ Total } & 2552 & 2643 & 3010 & & 2992 & \\
\hline
\end{tabular}

Fuente: $\quad$ Avelino, Esteban (2013). "Diagnóstico de Empleo y Remuneraciones en las Universidades Públicas (Caso UNMSM)" - Proyecto de investigación SIN - SIN.

\section{Tabla $N^{\circ} 7$}

Remuneración Mensual de Docentes de la UNMSM y de Magistrados del Poder Judicial. Año 2012

(Nuevos Soles)

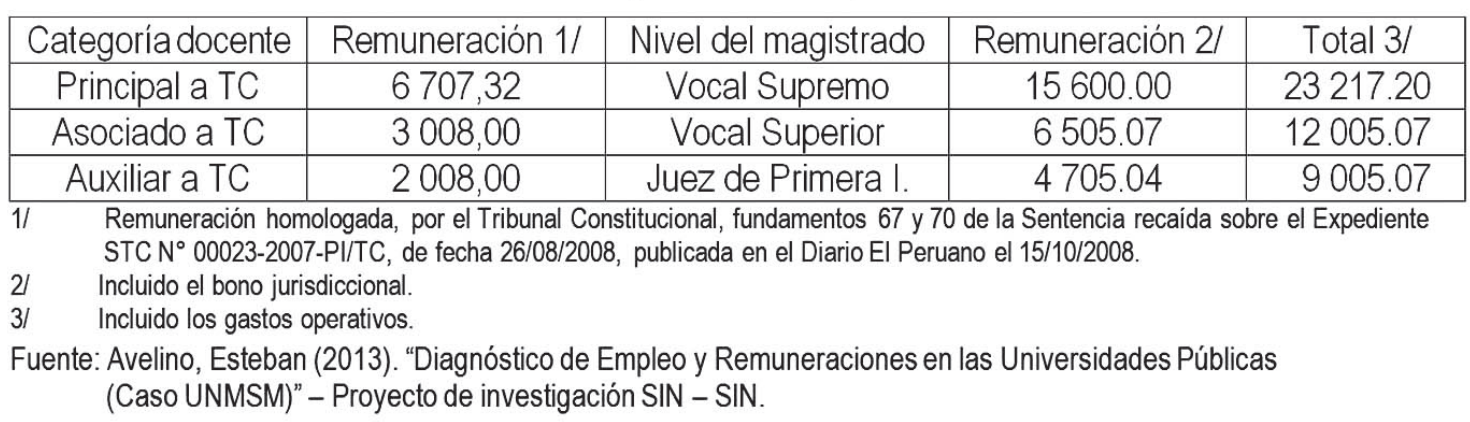

personales (más del 50\% del total de trabajadores no docentes) hasta el año 2009, han sido sustituidos por los contratos administrativos de servicios CAS. La remuneración mensual máxima de los funcionarios F-3 es inferior a S/. 1 750.00, de los Profesionales SPA inferior a S/.1500.00, de los Técnicos STA inferior a S/.1 300.00, de los Auxiliares SAA es inferior a S/.1 100.00; comparada con sus pares de Congreso, la remuneración mensual total de los trabajadores no docentes de la UNMSM representan menos del $20 \%$ de la remuneración mensual de los trabajadores del Congreso de grupos ocupacionales equivalentes.

\section{BENEFICIOS SOCIALES}

\section{Aguinaldo y bono por escolaridad}

Los docentes y trabajadores no docentes de la UNMSM, comprendidos en el Decreto Legislativo
276, de acuerdo con las Leyes 29626, 29812 y 29951 de Presupuesto del Sector Público, artículo $7^{\circ}$, numeral 7.1, literales a) y b) reciben un "aguinaldo" por "Fiestas Patrias" o "Navidad" de S/.300.00 y por bono de escolaridad una suma equivalente. Los trabajadores administrativos del Congreso y del Poder Judicial, comprendidos en el régimen laboral del Decreto Legislativo 728, reciben una gratificación por los mismos conceptos equivalente a una remuneración mensual habitualmente percibida: S/.5 000.00, S/. $10000.00, S / .15600 .00$, etc. Mientras los Docentes Principales de San Marcos reciben un "aguinaldo" por "Fiestas Patrias" o "Navidad" de S/. 300.00, los trabajadores de confianza del Congreso, del Poder Judicial y de otras reparticiones públicas con igual o menores méritos, por los mismos conceptos, reciben S/. 15600.00 (15 600 entre 300 son 52 veces más), una diferencia abismal. 


\section{Compensación por tiempo de servicios-CTS}

Base legal: Reglamento del Congreso, artículo $21^{\circ}$ y $22^{\circ}$; Ley del Poder Judicial, artículo $194^{\circ}$, Sentencia del TC y resoluciones del Poder Judicial; Decreto Legislativo 276 , artículo $54^{\circ}$, inciso c), modificado porla Ley N²5224; Decretos Legislativos 728 y 650. CTS de los congresistas. Remuneración adecuada, S/. 15 600.00; tiempo de servicios 5 años:

CTS $=15600.00 \times 5=S / .78000 .00$.

CTS de magistrados del Poder Judicial.

La Resolución Administrativa N 518-2007-GGPJ (del 19/07/2007) dispone que el cálculo de la CTS se hace incluyendo el bono por función jurisdiccional-BJ por separado, quedando condicionado el pago correspondiente al $\mathrm{BJ}$, a la asignación de recursos por el Gobierno Central.

Ejemplos:

CTS de Juez Supremo: Remuneración mensual, S/. 15 600.00; tiempo de servicios 30 años.

CTS $=15600.00 \times 5=$ S $/ .78000 .00$ ( 5 años de servicios, por curiosidad).

CTS $=15600.00 \times 30=S / .468000 .00$.

CTS de Juez Superior: Remuneración mensual, S/. 6 505.07; tiempo de servicios 25 años.

CTS $=6505.07 \times 5=\mathrm{S} / .32525 .35$ ( 5 años de servicios, por curiosidad).

CTS $=6505.07 \times 25=S / .162626 .75$.

CTS de Juez Primera Instancia: Remuneración mensual, S/. 4 705.04; Tiempo de servicios 20 años. CTS $=4705.04 \times 5=$ S/.23 525.20 ( 5 años de servicios, por curiosidad).

CTS $=4705.04 \times 20=$ S $/ .94100 .80$.

La mitad de la CTS de los magistrados jubilados o cesados a partir de 2007 estaría pendiente de pago.

CTS de docentes y no docentes de la UNMSM.

El inciso c) del artículo 54 del Decreto Legislativo 276, modificado por la Ley $\mathrm{N}^{\circ} 25224$, dice que la compensación por tiempo de servicios se otorga al personal nombrado al momento del cese por el importe del 50\% de su remuneración principal para los servidores con menos de 20 años de servicios o de una remuneración principal para los servidores con 20 o más años de servicios, por cada año completo o fracción mayor de 6 meses y hasta un máximo de 30 años de servicios.

Ejemplos:

CTS de Docente Principal: Remuneración principal, $S /$.39.37. Tiempo de servicios 30 años.

CTS $=39.37$ x $5=$ S/. 196.85 (5 años de servicios, por curiosidad).

$\mathrm{CTS}=39.37 \times 30=\mathrm{S} / .1181 .10$.

CTS de Docente Asociado: Remuneración principal, $S / .32 .23$. Tiempo de servicios 25 años.

CTS $=32.23 \times 5=S / .161 .15$ ( 5 años de servicios, por curiosidad).

CTS $=32.23 \times 25=S / .805 .75$.

CTS de Docente Auxiliar: Remuneración principal, S/.28.80. Tiempo de servicios 20 años.

CTS $=28.80 \times 5=S / .144 .00$ (5 años de servicios, por curiosidad).

$\mathrm{CTS}=28.80 \times 20=\mathrm{S} / .576 .00$.

CTS de funcionarios y trabajadores del sector público, comprendidos en el régimen laboral de la actividad privada" (Decretos Legislativos 728 y 650 ), puede ser adelantada hasta el $50 \%$ del monto previsto, para adquisición, construcción, reparación o remodelación de vivienda, amortización o pago de créditos hipotecarios.

Ejemplos:

CTS de un funcionario, con 20 años de servicios. Remuneración mensual S/. 15 000.00.

Remuneración computable $-\mathrm{RC}=15000+2500$ (un sexto de la gratificación por Fiestas Patrias o Navidad) = S/. 17500.00 .

CTS $=17500.00 \times 5=$ S/. 87500.00 (5 años de servicios, por curiosidad).

CTS $=17500.00 \times 20=S / .350000 .00$.

De conformidad con el TUO delDL 650, la CTS se deposita semestralmente en la empresa financiera 
LA DISPERSIÓN DE LOS REGÍMENES LABORALES EN EL SECTOR PÚBLICO LIMITAN LA REMUNERACIÓN HOMOLOGADA Y LOS BENEFICIOS DE LOS DOCENTES DE LA UNMSM

elegida por el trabajador; pero muchas entidades del sector público, con trabajadores comprendidos en el régimen laboral de la actividad privada no lo hacen, por lo que en la fecha de cese (o un tiempo después, vía procesos judiciales) los ex-trabajadores lo demandan y hacen pagar también intereses financieros, por obligaciones incumplidas; esta problemática muchos expertos lo ignoran.

\section{CONCLUSIONES}

1.- El 4 de julio de 2013 se publicó la Ley 30057, Ley del Servicio Civil, con la cual los sistemas remunerativos en el sector público suman 16 (sin incluir la ley de altos funcionarios del Estado), la que ha suscitado mucha oposición de sus trabajadores porque no resolvería el problema de fondo: la racionalidad de una escala remunerativa e igual remuneración para trabajos equivalentes, con ello los regímenes especiales no tendrían razón de existir.

2.- La "remuneración homologada" de los Docentes Principales de la UNMSM es menor al 30\% de la remuneración mensual total de los Jueces Supremos, en las categorías Asociado y Auxiliar, prácticamente, menor al $25 \%$ de la remuneración de los magistrados de los niveles con los cuales se homologó.

3.- Los Docentes Principales de la UNMSM reciben un "aguinaldo" por "Fiestas Patrias" o "Navidad" de $S / .300 .00$, los trabajadores de confianza del Congreso, del Poder Judicial y de otras reparticiones públicas con iguales o menores méritos, por los mismos conceptos, reciben S/. 15600.00 (15 600 entre 300 son 52 veces más), una diferencia abismal.

4.- La remuneración computable para cálculo de CTS de los Docentes Principales de la UNMSM, por cada año de servicios, es de $\mathrm{S} / .39 .37$, mientras que de los Jueces Supremos es de S/. 15 600.00; haciendo el cálculo por 30 de servicios resulta S/. 1181.10 y S/. 468 000.00, respectivamente. Si la comparación se hace con la remuneración computable para cálculo de CTS de los funcionarios de confianza, con méritos iguales que a los Docentes de las Universidades Públicas, la diferencia también es abismal.

\section{REFERENCIAS BIBLIOGRÁFICAS}

1. Comisión Multisectorial (creada por D.S. $\mathrm{N}^{\circ}$ 004-2001-TR). Informe sobre la Situación del Personal de la Administración Pública Central, publicado en Separata Especial en el Diario El Peruano: 25/07/2001.

2. Decreto Legislativo 276 (1984). Ley de Bases se la Carrera Administrativa y de Remuneraciones del Sector Público: Concordado.

3. Ley 23733 (1983). Ley Universitaria.

4. Decreto Supremo 017-93-JUS (1993). TUO de La Ley Orgánica del Poder Judicial.

\section{REFERENCIAS ELECTRÓNICAS}

1. GRADE (2006). Informe Final - Estudio sobre los Recursos Humanos en Salud en Perú: Gestión, Distribución, Normatividad, Regulación, Condiciones laborales y salariales y Formación. Página: http://www.grade.org.pe/download/docs/Informe\%20Final\%20RRHH\%20 Salud\%20GRADE.pdf.

2. SERVIR (MEF-Módulo de Gestión de Recursos Humanos, 2011). Los Regímenes Laborales en el Perú 2. http://files.servir.gob.pe/WWW/files/ biblioteca/SERVIR\%20-\%20El\%20servicio\%20 civil\%20peruano\%20-\%20Cap2.PDF.

3. Reglamento del Congreso de la República (i?). Texto actualizado. http://www.congreso.gob.pe/ accesible/reglamento/disp-finales.htm\#cuarta 